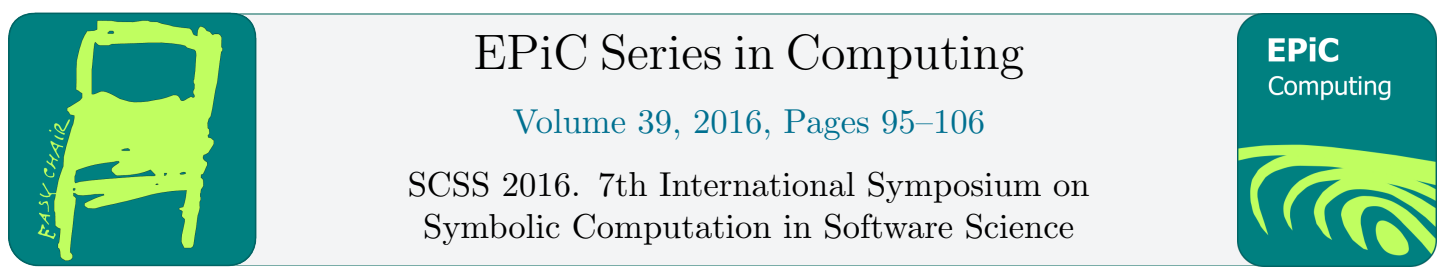

\title{
A Rewrite-based Computational Model for Functional Logic Programming
}

\author{
Mircea Marin ${ }^{1}$, Temur Kutsia ${ }^{2}$, and Besik Dundua ${ }^{3}$ \\ 1 Department of Computer Science \\ West University of Timişoara, Timişoara, Romania \\ mircea.marin@e-uvt.ro \\ 2 Research Institute for Symbolic Computation, \\ Johannes Kepler University, Linz, Austria \\ kutsia@risc.jku.at \\ 3 Institute of Applied Mathematics \\ Tbilisi State University \\ bdundua@gmail.com
}

\begin{abstract}
Functional logic programming is an extension of the functional programming style with two important capabilities: to define nondeterministic operations with overlapping rules, and to use logic variables in both defining rules and expressions to evaluate. A suitable model for functional logic programs are conditional constructor-based term rewrite systems (CB-CTRSs), which can be transformed into an equivalent program in a simpler class of rewrite systems (the core language) where computations can be performed more efficiently.

Recently, Antoy and Hanus proposed a translation of CB-CTRSs into an equivalent class of programs where computation can be performed efficiently by mere rewriting. Their computational model has the limitation of computing only ground answer substitutions for equations with strict semantics interpreted as joinability to a value. We propose two adjustments of their computational models, which are capable to compute non-ground answers.
\end{abstract}

\section{Introduction}

Functional logic programming (FLP for short) is a declarative programming style resulted from the successful amalgamation of the best features of functional programming with logic programming (see [7] for a recent survey). In comparison with pure functional languages, functional logic languages have more expressive power due to the possibility to define nondeterministic operations with overlapping rules (i.e., more than one rule can be applied to evaluate a function call), and the possibility to use logic variables in both defining rules and expressions to evaluate.

The presence of logic variables makes the evaluation of an expression $t$ in the presence of a program $\mathcal{R}$ more challenging: We are not only interested to compute a value to which $t$ reduces (that is, a ground constructor term $v$ such that $t \rightarrow_{\mathcal{R}}^{*} v$ ), but rather to compute a set of pairs $\langle\sigma, v\rangle$ made of a ground constructor substitution $\sigma$ for the variables of $t$ and a value $v$, such that 
$t \sigma \rightarrow_{\mathcal{R}}^{*} v$. We may denote the set of all such pairs by $\operatorname{Sol}_{\mathcal{R}}(t)$ and call its elements solutions of $t$. A convenient representation of $\operatorname{Sol}_{\mathcal{R}}(t)$ is by a set $A$ of so called computed answers, with the following two properties: (1) $A \subseteq \operatorname{Sol}_{\mathcal{R}}(t)$ (soundness), and (2) for every solution $\langle\varphi, v\rangle$ of $t$ there exist $\langle\sigma, v\rangle \in A$ and a constructor substitution $\eta$ such that $\varphi(x)=\eta(\sigma(x))$ for every variable $x$ in $t$ (completeness).

The computational models of FLP languages are designed to compute sound and complete sets of computed answers. When the interest is in solving systems of equations, we can model them by terms of the form

$$
e_{1} \& \& \ldots \& e_{n}
$$

where every $e_{i}$ is an equational term with a certain semantics, and \&\&/2 is a predefined rightassociative infix operation. In general, equational terms are of the form $s \approx t$, and defined to hold if $s$ and $t$ are reducible to the same (ground) constructor term. The nice thing when interpreting $\approx$ as joinability to a value is that we can extend $\mathcal{R}$ with rewrite rules for $\approx / 2$ and \&\&/2, and obtain a TRS $\mathcal{R}_{\text {eq }}$ with the following property: $\sigma$ is a solution of an equational goal $G=s_{1} \approx t_{1} \& \& \ldots \& \& s_{n} \approx t_{n}$ iff $\sigma$ is a constructor substitution and $G \sigma \rightarrow_{\mathcal{R}_{\text {eq }}}^{*}$ success. (See, e.g., [3].) This observation leads to the possibility to solve such systems of equations by narrowing, a mechanism that extends the concept of reduction from functional programming with unification and nondeterministic search from logic programming. Narrowing was designed initially as a sound and complete method for solving unification problems in equational theories presented by confluent term rewrite systems [10]:

If $\mathcal{R}$ is such a TRS and $\mathcal{R}^{\prime}=\mathcal{R} \cup\{x \approx x \rightarrow$ success $\}$, then narrowing w.r.t. $\mathcal{R}^{\prime}$ computes a complete set of $\mathcal{R}$-unifiers of $s$ and $t$.

However, this way of computing answers is largely useless and hopelessly inefficient for FLP, because:

1. Unrestricted narrowing computes many $\mathcal{R}$-unifiers which are not solutions from the point of view of FLP.

2. $\mathcal{R}^{\prime}$ ensures the interpretation of equality as joinability to any term. Nowadays, most FLP languages interpret equality as joinability to a common (ground) constructor term.

3. The search space of narrowing explores the possibility to unify each rule with each nonvariable subterm of the input system. The resulting search space would be huge even for small TRSs.

Therefore, many narrowing strategies and narrowing calculi have been proposed, to reduce the search space for solutions without losing the completeness of the set of computed answers.

- Narrowing strategies are partial mappings from terms to narrowing steps, and their rôle is to tell us which subterms to select for narrowing, in order to compute a sound and complete set of answer substitutions.

- Narrowing calculi emerged as an alternative to narrowing strategies: Usually, they consist of a small set of elementary inference or transformation rules for equational goals, which can be used to simulate narrowing steps. Typical examples of narrowing calculi are LNC [15], $\mathrm{LNC}_{\mathrm{d}}$ [14], LCNC [8], LCNC [16], and $\mathrm{LCNC}_{\mathrm{d}}$ [13]. Higher-order versions of narrowing calculi are also known, e,g., LN [17], HOLN [11], and $\mathrm{LN}_{\mathrm{ff}}$ [12].

The success of finding an adequate strategy or calculus for narrowing depends mainly on the class of TRSs chosen to represent programs, and on the way we define equality and the admissibility of solutions. Several interesting sound and complete narrowing strategies are already known for various classes of first-order left linear constructor-based TRS (CB-TRSs for short). 
To this category belong the following strategies: needed narrowing [4], for strongly sequential TRSs [9], parallel narrowing [5], for weakly orthogonal TRSs, and the INS narrowing strategy [2] for overlapping inductively sequential TRSs.

Programs presented by conditional CB-TRSs (CB-CTRSs for short) are desirable because they increase the conciseness and expressive power of rule-based definitions of operations of interest. In [6], Antoy and Hanus report a rewrite-based computational model for FLP with CB-CTRSs. Their main results can be summarised as follows:

1. They identify a transformation of CB-CTRSs into equivalent overlapping inductively sequential TRSs with extra variables (OISs for short). OISs are a simpler class of TRSs, for which we already know a sound and complete narrowing strategy [2].

2. They identify a sound and complete computational model for FLP for programs presented by OIS, based on a translation of OIS with extra variables into OIS without extra variables, and on rewriting of term/substitution pairs.

These results open the possibility to produce an efficient implementation of a sound and complete narrowing strategy for FLP with CB-CTRSs, by reducing all computations to rewrites of term/substitution pairs with respect to OISs. There are, however, some important limitations:

1. Equality is interpreted as reducibility to a common value.

2. The computed answers are ground constructor substitutions. As a result, the completeness property of the calculus implies that the set of computed answers is often infinite, and thus the solving process of a system of equations runs forever.

In this paper we overcome these limitations and propose a rewrite-based strategy for functional logic programming with programs presented by OISs. We argue that this strategy is sound and complete in the general sense, where we do not restrict ourself to ground solutions, and interpret equality as reducibility to a common, not necessarily ground, constructor term.

The paper is structured as follows. Section 2 reviews concepts and notations used in this paper, and previous results with relevance for our investigation. Sections 3 and 4 describe the strategies proposed by us for solving systems of equations when programs are presented by OISs and equality is interpreted in the more general sense, mentioned above. The first one is a narrowing strategy obtained by an adjustment of the INS strategy, and the second one is a rewrite-based strategy. Both of them are sound and complete. Section 5 concludes.

\section{Preliminaries}

We consider a finite many-sorted signature $\Sigma$ partitioned into a set $\mathcal{C}$ of (data) constructors, and a set $\mathcal{F}$ of (defined) function symbols or operations. We write $f / n$ to indicate the fact that $f \in \Sigma$ is an $n$-ary constructor or operation. We also consider $\mathcal{X}$ to be a countably infinite set of sorted variables. The sets $\mathcal{T}(\Sigma, \mathcal{X}), \mathcal{T}(\mathcal{C}, \mathcal{X})$ and $\mathcal{T}(\mathcal{C})$ of well-sorted terms, constructor terms, and ground constructor terms are defined as usual. The terms of $\mathcal{T}(\mathcal{C})$ are also called values.

We write $\operatorname{var}(t)$ for the set of variables which occur in a term $t$. A term $t$ is ground if $\operatorname{var}(t)=\emptyset$, and linear if it does not contain multiple occurrences of a variable. A pattern is a term $f\left(t_{1}, \ldots, t_{n}\right)$ where $f / n \in \mathcal{F}$ and $t_{1}, \ldots, t_{n} \in \mathcal{T}(\mathcal{C}, \mathcal{X})$.

Next, we introduce equations and systems of equations: Their syntax is defined in the extended signature $\Sigma_{\text {eq }}:=\Sigma \cup\{\approx, \& \&, \doteq\}$ where $\approx / 2, \doteq / 2$ and \&\&/2 are new operations to which we will give a special interpretation. An equation is a term of the form $\approx(s, t)$ or $\doteq(s, t)$, where $s, t \in \mathcal{T}(\Sigma, \mathcal{X})$ are terms of same sort. A system of equations is either an equation or a 
term \&\& $(e, c)$ where $e$ is an equation and $c$ is a system of equations. To improve readability, we will use the following notations and assumptions:

- We write equations and systems of equations in infix notation, that is, we write $s \approx t$ instead of $\approx(s, t), s \doteq t$ instead of $\doteq(s, t)$, and $e \& \& c$ instead of \&\& $(e, c)$.

- We assume that \&\&/2 is right-associative, and write $e_{1} \& \& e_{2} \& \& \ldots \& \& e_{n-1} \& \& e_{n}$ instead of $\& \&\left(e_{1}, \& \&\left(e_{2}, \ldots, \& \&\left(e_{n-1}, e_{n}\right) \ldots\right)\right)$.

A rewrite rule is an expression of the form $l \rightarrow r$ where $l$ is a pattern and $r$ is a term of the same sort as $l$. A conditional rewrite rule is of the form $l \rightarrow r \Leftarrow c$ where $l, r$ satisfy the same conditions as before, and $c$ is a system of equations, called the conditional part of the rule. The set of extra variables of $l \rightarrow r$ (resp. $l \rightarrow r \Leftarrow c$ ) is evar $(l \rightarrow r):=\operatorname{var}(r) \backslash \operatorname{var}(l)$ (resp. $\operatorname{evar}(l \rightarrow r \Leftarrow c):=(\operatorname{var}(r) \cup \operatorname{var}(c)) \backslash \operatorname{var}(l))$.

In functional logic programming, programs are modelled by finite sets of rewrite rules, which may be conditional. In theory, a set of such rewrite rules is called constructor-based term rewrite system (CB-TRS). Finite sets which may also contain conditional rewrite rules are called conditional constructor-based TRSs (CB-CTRSs).

To formally define computations with respect to a given program (that is, CB-CTRS), we shall introduce a few more notions. Positions in a term are denoted by sequences of natural numbers. We write $\left.t\right|_{p}$ for the subterm of $t$ at position $p$, and $t[s]_{p}$ for the result of replacing $\left.t\right|_{p}$ with $s$ at position $p$ in $t$. A substitution is a mapping $\sigma: \mathcal{X} \rightarrow \mathcal{T}(\Sigma, \mathcal{X})$ such that its domain $\operatorname{dom}(\sigma):=\{x \in \mathcal{X} \mid \sigma(x) \neq x\}$ is finite, and $x$ and $\sigma(x)$ are of the same sort for every $x \in \mathcal{X}$. It is common practice to denote a substitution $\sigma$ by the finite set $\{x \rightarrow \sigma(x) \mid x \in \operatorname{dom}(\sigma)\}$. The restriction of a substitution $\sigma$ to a set of variables $V \subseteq \mathcal{X}$ is the substitution $\left.\sigma\right|_{V}$ with $\operatorname{dom}\left(\left.\sigma\right|_{V}\right)=\operatorname{dom}(\sigma) \cap V$ and $\left.\sigma\right|_{V}(x)=\sigma(x)$ for all $x \in \operatorname{dom}\left(\left.\sigma\right|_{V}\right)$. A (ground) constructor substitution is a substitution $\sigma$ such that $\sigma(x)$ is a (ground) constructor term for every $x \in$ $\operatorname{dom}(\sigma)$. Substitutions are extended to morphisms on terms in the obvious way, and we write $t \sigma$ for the image of $t$ under the morphism induced by a substitution $\sigma$. Subsumption is the ordering on terms defined by $s \leq t$ if $s \sigma=t$ for some substitution $\sigma$. Also, we write $s<t$ if $s \leq t$ and $t \not \leq s$. The subsumption relation is defined on substitutions too: $\sigma \leq \sigma^{\prime}$ if there exists a substitution $\eta$ such that $\sigma^{\prime}=\left.(\eta \circ \sigma)\right|_{\operatorname{vars}\left(\sigma^{\prime}\right)}$. A variant of $t$ is $t^{\prime}$ such that $t \leq t^{\prime}$ and $t^{\prime} \leq t$. It is well known that $t$ is a variant of $t^{\prime}$ if and only if there exists a bijective substitution $\sigma: \mathcal{X} \rightarrow \mathcal{X}$ such that $t^{\prime}=t \sigma . t^{\prime}$ is a fresh variant of $t$ if it is a variant of $t$ and $\operatorname{var}\left(t^{\prime}\right)$ consists of variables which did not occur in the expressions encountered so far. A unifier of two terms $s$ and $t$ is a substitution $\sigma$ such that $s \sigma=t \sigma$. A unifier $\sigma$ is a most general unifier ( $m g u$ for short) if $\sigma \leq \sigma^{\prime}$ for any other unifier $\sigma^{\prime}$ of $s$ and $t$.

A rewrite step of a term $s$ with respect to a CB-TRS $\mathcal{R}$ is a relation $s \rightarrow_{p, l \rightarrow r, \sigma} s^{\prime}$, which is defined if there exist a position $p$ in $s$, a rewrite rule $l \rightarrow r \in \mathcal{R}$ with a fresh variant $l^{\prime} \rightarrow r^{\prime}$, and a substitution $\sigma$ such that $\left.t\right|_{p}=l^{\prime} \sigma$ and $s^{\prime}=s\left[r^{\prime} \sigma\right]_{p}$. We may write $s \rightarrow_{\mathcal{R}} s^{\prime}$ instead of $s \rightarrow_{p, l^{\prime} \rightarrow r^{\prime}, \sigma} s^{\prime}$. In general, if $\rightarrow$ is a binary relation on terms, we may also refer to the following relations induced by $\rightarrow$ :

- the reflexive-transitive closure of $\rightarrow: s \rightarrow^{*} t$ if $s \rightarrow^{n} t$ for some $n \in \mathbb{N}$, where

$$
\begin{aligned}
& s \rightarrow^{0} t \text { if } s=t, \text { and } \\
& s \rightarrow^{n+1} t \text { if } s \rightarrow u \text { and } u \rightarrow^{n} t \text { for some term } u .
\end{aligned}
$$

- strict joinability: $s \downarrow t$ if $s \rightarrow^{*} u$ and $t \rightarrow^{*} u$ for some $u \in \mathcal{T}(\mathcal{C}, \mathcal{X})$.

- strict joinability to a value: $s \rrbracket t$ if $s \rightarrow^{*} v$ and $t \rightarrow^{*} v$ for some $v \in \mathcal{T}(\mathcal{C})$.

The joinability relations $\downarrow_{\mathcal{R}}$ and $\bigvee_{\mathcal{R}}$ are used to interpret the equality operations $\approx / 2$ and $\doteq / 2$ with respect to a CB-TRS $\mathcal{R}$ : We say that $s \approx t$ (resp. $s \doteq t$ ) holds with respect to $\mathcal{R}$ if $s \downarrow_{\mathcal{R}} t$ 
holds (resp. $s \coprod_{\mathcal{R}} t$ holds). More generally, a system of equations $c$ is said to hold with respect to $\mathcal{R}$ if every component equation of $c$ holds with respect to $\mathcal{R}$.

The interpretations of $\approx / 2$ and $\doteq / 2$ remain the same when $\mathcal{R}$ is a CB-CTRS, whereas the rewrite relation $\rightarrow_{\mathcal{R}}$ is generalized as follows: $s \rightarrow_{\mathcal{R}} s$ if there exist a non-variable position $p$ in $t$, a rule $l \rightarrow r \Leftarrow c$ from $\mathcal{R}$ with fresh variant $l^{\prime} \rightarrow r^{\prime} \Leftarrow c^{\prime}$, and a substitution $\sigma$ such that

- $\left.s\right|_{p}=l^{\prime} \sigma, s^{\prime}=s\left[r^{\prime} \sigma\right]_{p}$, and

- $c^{\prime} \sigma$ holds w.r.t. $\mathcal{R}$, that is: $t \sigma \downarrow_{\mathcal{R}} t^{\prime} \sigma$ for all $t \approx t^{\prime}$ in $c$, and $t \sigma \searrow_{\mathcal{R}} t^{\prime} \sigma$ for all $t \doteq t^{\prime}$ in $c$.

In this case, we may write $s \rightarrow_{p, l \rightarrow r \Leftarrow c, \sigma} s^{\prime}$ instead of $s \rightarrow_{\mathcal{R}} s^{\prime}$.

Let $G$ be a system of equations. A substitution $\sigma$ is a solution of $G$ w.r.t. $\mathcal{R}$ if $\sigma$ is a constructor substitution and $G \sigma$ holds w.r.t. $\mathcal{R}$. A complete set of answers for $G$ is a set $A$ of solutions for $G$ such that, for every solution $\sigma^{\prime}$ of $G$ there exists $\sigma \in A$ such that $\sigma \leq \sigma^{\prime}$.

A narrowing step of a term $s$ with respect to a CB-CTRS $\mathcal{R}$ is a relation $s \rightsquigarrow \mathcal{R}, \sigma s^{\prime}$ which is defined to hold if there exist a non-variable position $p$ of $s$ and a rule $l \rightarrow r \Leftarrow c$ from $\mathcal{R}$ with fresh variant $l^{\prime} \rightarrow r^{\prime} \Leftarrow c^{\prime}$, such that $s \sigma \rightarrow_{p, l^{\prime} \rightarrow r^{\prime} \Leftarrow c^{\prime}, \sigma} s^{\prime}$ (i.e., $\left.s\right|_{p} \sigma=l^{\prime} \sigma, s^{\prime}=s\left[r^{\prime} \sigma\right]_{p}$, and $c^{\prime} \sigma$ holds w.r.t. $\mathcal{R}$ ). A sequence $s \rightsquigarrow \mathcal{R}, \sigma_{1} s_{1} \rightsquigarrow \mathcal{R}, \sigma_{2} \ldots \rightsquigarrow \mathcal{R}, \sigma_{n} s_{n}$ of narrowing steps is abbreviated $s \rightsquigarrow n_{\mathcal{R}, \sigma}^{n} s_{n}$, or simply $s \rightsquigarrow{ }_{\mathcal{R}, \sigma}^{*} s_{n}$, where $\sigma=\sigma_{n} \circ \ldots \circ \sigma_{2} \circ \sigma_{1}$.

The evaluation of $s$ in an FP language presented by a CB-CTRS $\mathcal{R}$ yields a value $v \in \mathcal{T}(\mathcal{C})$ such that $s \rightarrow_{\mathcal{R}}^{*} v$, whereas the evaluation of $s$ in FLP is concerned with computing a set $A n s_{\mathcal{R}}(s)$ of pairs $\langle v, \sigma\rangle$ with $v \in \mathcal{T}(\mathcal{C})$ and $\sigma$ a constructor substitution, such that $s \sigma \rightarrow_{\mathcal{R}}^{*} v$ (soundness). In addition, we also wish the set $\operatorname{Ans}_{\mathcal{R}}(s)$ to be complete in the following sense:

For any constructor substitution $\sigma^{\prime}$ such that $s \sigma^{\prime} \rightarrow_{\mathcal{R}}^{*} v$, there exists $\langle v, \sigma\rangle \in A n s_{\mathcal{R}}(t)$ such that $\sigma \leq \sigma^{\prime}$.

Narrowing-based computational models compute the elements $\langle v, \sigma\rangle$ of $A n s_{\mathcal{R}}(s)$ by producing a set of narrowing derivations $s \rightsquigarrow_{\mathcal{R}, \sigma}^{*} v$ with constructor substitution $\sigma$ and value $v$. Soundness follows from the observation that $s \rightsquigarrow_{\mathcal{R}, \sigma}^{*} v$ implies $s \sigma \rightarrow_{\mathcal{R}}^{*} v$. Computational efficiency can be often achieved by using a narrowing strategy, which prescribes, for every term, what narrowing step(s) to perform next, without losing completeness. Formally, a narrowing strategy is a partial function $\mathcal{S}$ on terms such that, for amy term $t, \mathcal{S}(t)$ is either undefined or a set of triples $\langle p, \rho, \sigma\rangle$ with constructor substitution $\sigma$ such that we can perform the narrowing step $t \rightsquigarrow p, \rho, \sigma t^{\prime}$. The evaluation of a term $s$ with $\mathcal{S}$ yields the set of computed answers

$$
\begin{aligned}
A n s_{\mathcal{R}}^{\mathcal{S}}(s)=\{\langle v, \sigma\rangle \mid & s \rightsquigarrow_{\mathcal{R}, \sigma}^{*} v \text { where } v \in \mathcal{T}(\mathcal{C}) \text { and }(p, \rho, \sigma) \in \mathcal{S}(t) \\
& \text { for every narrowing step } t \rightsquigarrow p, \rho, \sigma \\
& \left.t^{\prime} \text { of } s \rightsquigarrow_{\mathcal{R}, \sigma}^{*} v\right\} .
\end{aligned}
$$

\section{Overlapping inductively sequential rewrite systems}

We define here the class of CB-TRSs that concerns us most: overlapping inductively sequential TRSs with extra variables. They are defined via an auxiliary hierarchical data structure, called definitional tree. The following definitions are adapted from $[1,2]$.

A partial definitional tree ( $p d t)$ of a linear pattern $t$ is a tree-like structure $\mathcal{T}$ with nodes of one of the following three forms:

1. $\operatorname{branch}\left(t, p, \mathcal{T}_{1}, \ldots, \mathcal{T}_{k}\right)$ where $\left.t\right|_{p}$ is a variable of a sort $s$ with constructors $c_{1} / n_{1}, \ldots, c_{k} / n_{k}$ in standard ordering, and every $\mathcal{T}_{i}(1 \leq i \leq k)$ is a pdt of the pattern $t\left[c_{i}\left(x_{1}, \ldots, x_{n_{i}}\right)\right]_{p}$ and $x_{1}, \ldots, x_{n_{i}}$ are distinct fresh variables of appropriate sorts. The patterns $t\left[c_{i}\left(x_{1}, \ldots, x_{n_{i}}\right)\right]_{p}$ are called the children of $t$ in the pdt, and $t$ their parent. Also, the variable $\left.t\right|_{p}$ is referred to as inductive variable of $t$. 
2. $r u l e\left(t, t \rightarrow r_{1} ? \ldots ? r_{k}\right)$ where $t \rightarrow r_{1}, \ldots, t \rightarrow r_{k}$ are distinct variants of rewrite rules from $\mathcal{R}$. We say about this node that it contains the rule variants $t \rightarrow r_{1}, \ldots, t \rightarrow r_{k}$.

Note that the expression $t \rightarrow r_{1}$ ? $\ldots ? r_{k}$ is not a rule, but a compact abbreviation of the set of rules $\left\{t \rightarrow r_{1}, \ldots, t \rightarrow r_{k}\right\}$, where the symbol '?' is a separator between the alternative right-hand sides of the existing rules.

3. exempt $(t)$.

The first argument of every node is called the pattern of that node, and the set of patterns of a pdt consists of the patterns of its nodes. A definitional tree of an operation $f / n$ is a pdt of a pattern $f\left(x_{1}, \ldots, x_{n}\right)$ where $x_{1}, \ldots, x_{n}$ are distinct variables. An operation $f$ of $\mathcal{R}$ is overlapping inductively sequential if there exists a definitional tree $\mathcal{T}$ of $f$ whose rule-nodes contain a variant for every rule which defines $f$ in $\mathcal{R}$. A CB-TRS is overlapping inductively sequential (OIS for short) if every operation $f$ is overlapping inductively sequential.

For example, the CB-TRS

$$
\begin{aligned}
\mathcal{R}=\{ & \operatorname{ins}(x, \operatorname{null}) \rightarrow \operatorname{cons}(x, \operatorname{null}), \\
& \operatorname{ins}(x, \operatorname{cons}(y, z)) \rightarrow \operatorname{cons}(x, \operatorname{cons}(y, z)), \\
& \operatorname{ins}(x, \operatorname{cons}(y, z)) \rightarrow \operatorname{cons}(y, \operatorname{ins}(x, z))\}
\end{aligned}
$$

is OIS because its function symbol ins has the definitional tree

$$
\begin{aligned}
& \mathcal{T}_{\text {ins }}=\operatorname{branch}(\operatorname{ins}(x, y), 2 \text {, } \\
& \text { rule }(\operatorname{ins}(x, \text { null }) \rightarrow \operatorname{cons}(x, \text { null })) \text {, } \\
& \left.\operatorname{rule}\left(\operatorname{ins}\left(x, \operatorname{cons}\left(y_{1}, z_{1}\right)\right) \rightarrow \operatorname{cons}\left(x, \operatorname{cons}\left(y_{1}, z_{1}\right)\right) ? \operatorname{cons}\left(y_{1}, \operatorname{ins}\left(x, z_{1}\right)\right)\right)\right)
\end{aligned}
$$

It is obvious that every OIS is a left-linear CB-TRS, and every operation-rooted term $f\left(t_{1}, \ldots, t_{n}\right)$ is unifiable with some patterns from a definitional tree of $f$.

A sound and complete narrowing strategy for OIS is inductively sequential narrowing (INS): for every term $t$, it computes a (possibly empty) set $I N S_{\mathcal{R}}(t)$ of triples $\langle p, l \rightarrow r, \sigma\rangle$ which indicate the need to perform the narrowing step $t \rightsquigarrow p, l \rightarrow r, \sigma t^{\prime}$, which has the same effect as the rewrite step $t \sigma \rightarrow_{p, l \rightarrow r} t^{\prime}$. This strategy assumes fixed a set $\left\{\mathcal{T}_{f} \mid f \in \mathcal{F}\right\}$ of definitional trees for all operations in $\mathcal{F}$, and is defined as follows:

1. If $t=f\left(t_{1}, \ldots, t_{n}\right)$ is an operation-rooted term, then $I N S_{\mathcal{R}}(t)=\varphi_{\mathcal{R}}\left(t, \mathcal{T}_{f}\right)$ where $\varphi_{\mathcal{R}}(t, \mathcal{T})$ is defined by case distinction on the type of $\mathcal{T}$ :

(a) If $\mathcal{T}=\operatorname{branch}\left(\pi, p, \mathcal{T}_{1}, \ldots, \mathcal{T}_{k}\right)$, let $s$ be the sort of $\left.t\right|_{p}$ and $c_{1} / n_{1}, \ldots, c_{k} / n_{k}$ be the data constructors of sort $s$ in canonical order. We distinguish 3 subcases:

i. If $\left.t\right|_{p}=x \in \mathcal{X}$, let $\sigma_{i}=\left\{x \rightarrow c_{i}\left(x_{1}, \ldots, x_{n_{i}}\right)\right\}$ be substitutions with $x_{1}, \ldots, x_{n_{i}}$ fresh variables of appropriate sorts, for all $1 \leq i \leq n$.

Then $\varphi_{\mathcal{R}}(t, \mathcal{T})=\bigcup_{i=1}^{k}\left\{\left\langle q, l \rightarrow r, \sigma_{i} \circ \eta_{i}\right\rangle \mid\left\langle q, \bar{l} \rightarrow r, \eta_{i}\right\rangle \in \varphi_{\mathcal{R}}\left(t \sigma_{i}, \mathcal{T}_{i}\right)\right\}$.

ii. If $\left.t\right|_{p}=c_{i}\left(t_{1}, \ldots, t_{n_{i}}\right)$ then $\varphi_{\mathcal{R}}(t, \mathcal{T})=\varphi_{\mathcal{R}}\left(t, \mathcal{T}_{i}\right)$.

iii. Otherwise, the root of $\left.t\right|_{p}$ is an operation. In this case

$$
\varphi_{\mathcal{R}}(t, \mathcal{T})=\left\{\langle p \cdot q, l \rightarrow r, \eta\rangle \mid\langle q, l \rightarrow r, \eta\rangle \in I N S_{\mathcal{R}}\left(\left.t\right|_{p}\right)\right\} .
$$

(b) If $\mathcal{T}=\operatorname{rule}\left(\pi, \pi \rightarrow r_{1}\right.$ ? $\ldots$ ? $\left.r_{k}\right)$ then $\varphi_{\mathcal{R}}(t, \mathcal{T})=\left\{\left\langle\lambda, \pi \rightarrow r_{i}, \emptyset\right\rangle \mid 1 \leq i \leq k\right\}$.

(c) If $\mathcal{T}=\operatorname{exempt}(\pi)$ then $\varphi_{\mathcal{R}}(t, \mathcal{T})=\emptyset$.

2. If $t \in \mathcal{X}$ then $\varphi_{\mathcal{R}}(t, \mathcal{T})=\emptyset$, 
3. Otherwise, $t=c\left(t_{1}, \ldots, t_{n}\right)$ with $c / n$ a constructor symbol. In this case

$$
I N S_{\mathcal{R}}(t)= \begin{cases}\emptyset & \text { if } t \in \mathcal{T}(\mathcal{C}, \mathcal{X}), \\ \left\{\langle i \cdot q, l \rightarrow r, \sigma\rangle \mid\langle q, l \rightarrow r, \sigma\rangle \in I N S_{\mathcal{R}}\left(t_{i}\right)\right\} & \text { if } i=\min \left\{j \mid I N S_{\mathcal{R}}\left(t_{j}\right) \neq \emptyset\right\} .\end{cases}
$$

INS can also be used to solve systems of strict equations $G=s_{1} \doteq t_{1} \& \& \ldots \& \& s_{n} \doteq t_{n}$ w.r.t. an OIS $\mathcal{R}$. To achieve this, we can extend the signature $\Sigma$ with the operations \&\& $/ 2, \doteq / 2$ and the constructor success $/ 0$, and extend $\mathcal{R}$ to the OIS $\mathcal{R}_{\text {eq }}=\mathcal{R} \cup\left\{\rho_{c} \mid c \in \mathcal{C}\right\} \cup\{$ success \&\& $x \rightarrow x\}$, where every rewrite rule $\rho_{c}$ is of the form

$$
c\left(x_{1}, \ldots, x_{n}\right) \doteq c\left(y_{1}, \ldots, y_{n}\right) \rightarrow \text { success } \& \& x_{1} \doteq y_{1} \& \& \ldots \& \& x_{n} \doteq y_{n}
$$

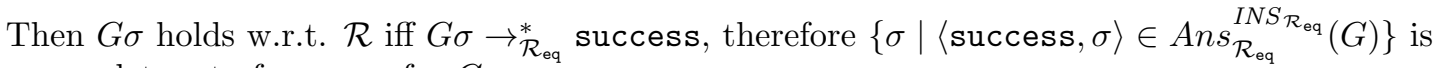
a complete set of answers for $G$.

\section{Well-known results}

Finally, we recall the theoretical results already mentioned in the Introduction, which represented the starting point of our further investigation:

1. Every CB-CTRS $\mathcal{R}$ where the conditional parts of rules are equational systems of the form

$$
s_{1} \doteq t_{1} \& \& \ldots \& \& s_{n} \doteq t_{n}
$$

can be transformed into an equivalent OIS [3], in two stages:

- Linearization: Every rule $l \rightarrow r \Leftarrow c$ where $l$ is a nonlinear pattern is transformed into $l^{\prime} \rightarrow r \Leftarrow c \& \& c^{\prime}$ where $l^{\prime}$ is obtained from $l$ by replacing all its variable occurrences with distinct fresh variables, and $c^{\prime}$ is a conjunction of equations of the form $x \doteq y$ which express the fact that $y$ is the fresh variable that replaced an occurrence of variable $x$ in $l$. For example, the linearization of

$$
f(c(x, c(x, y))) \rightarrow z \Leftarrow f(c(x, y)) \doteq z
$$

is

$$
f\left(c\left(x_{1}, c\left(x_{2}, x_{3}\right)\right)\right) \rightarrow z \Leftarrow f(c(x, y)) \doteq z \& \& x_{1} \doteq x \& \& x_{2} \doteq y \& \& x_{3} \doteq z .
$$

- Deconditionalization: Every left-linear conditional rewrite rule $l \rightarrow r \Leftarrow c$ is transformed into the unconditional rule $l \rightarrow$ if $(c, r)$ over the signature extended with the operation if $/ 2$. To properly interpret the if $/ 2$, we add the rule if (success, $x) \rightarrow x$.

This means that, if $\mathcal{R}$ is a CB-CTRS whose rules satisfy syntactic restriction (3), and $\overline{\mathcal{R}}$ is the OIS with extra variables produced by the transformation mentioned above, then $A N S_{\overline{\mathcal{R}}}^{I N S_{\overline{\mathcal{R}}}}(s)$ is a sound and complete set of computed answers of the term $s$ w.r.t. $\mathcal{R}$. Strategy INS can be used to compute complete sets of computed answers for systems of strict equations too: If $\mathcal{R}$ is a CB-CTRS and $G=s_{1} \doteq t_{1} \& \& \ldots \& \& s_{n} \doteq t_{n}$ is such a system of equations, then

$$
\left\{\sigma \mid\langle\sigma, \text { success }\rangle \in A N S_{\overline{\mathcal{R}}_{\text {eq }}}^{I N S_{\overline{\mathcal{R}}_{\mathrm{eq}}}}(G)\right\}
$$

is a complete set of computed answers of $G$ with respect to $\mathcal{R}$. 
2. The authors of [6] propose a mapping $X E P$ that transforms an OIS with extra variables $\mathcal{R}$ into a set $X E P(\mathcal{R})$ of rules $l \rightarrow\langle r, \psi\rangle$ from terms to term/substitution pairs, and a term $t$ into a term/substitution pair $\langle t, \chi\rangle$. Also, they identify a rewrite relation $\rightarrow_{\mathcal{R}^{\prime}}$ induced by $\mathcal{R}^{\prime}=X E P(\mathcal{R})$ between term/substitution pairs, such that $\left\{\langle v, \sigma\rangle \mid X E P(t) \rightarrow_{\mathcal{R}^{\prime}}^{*}\langle v, \sigma\rangle\right\}$ is a sound and complete set of answers for $t$.

A somewhat unexpected consequence of this result is that the power of narrowing computations specific to functional logic programming can be obtained by mere rewriting.

\section{Dropping the restriction to groundness}

The results mentioned so far have been proved for the case when equality is interpreted as joinability to a value. This means, all equational terms in goals and the conditional parts of rewrite rules, are of the form $s \doteq t$. In this section we analyse what happens if drop the restriction to groundness, that is, if we interpret equality as joinability to a constructor term.

Suppose $\mathcal{R}$ is a CB-CTRS where all equations in the conditional parts are of the form $s \doteq t$, and $\operatorname{tr}(\mathcal{R})$ is the CB-CTRS produced by replacing all occurrences of $\doteq / 2$ with $\approx / 2$. Since every value is a constructor term, the following statements hold trivially:

$$
\text { If } s \rightarrow_{\mathcal{R}} s^{\prime} \text { then } s \rightarrow_{\operatorname{tr}(\mathcal{R})} s^{\prime} \text {. If } s \downarrow_{\mathcal{R}} s^{\prime} \text { then } s \coprod_{\operatorname{tr}(\mathcal{R})} s^{\prime} .
$$

For the converse, we have the following lemma.

Lemma 1. Let $s, s^{\prime}$ be terms, and $\theta$ be a value substitution with $\operatorname{var}(s) \cup \operatorname{var}\left(s^{\prime}\right) \subseteq \operatorname{dom}(\theta)$. The following statements hold:

1. If $s \rightarrow_{\operatorname{tr}(\mathcal{R})} s^{\prime}$ then $s \theta \rightarrow_{\mathcal{R}} s^{\prime} \theta$.

2. If $s \coprod_{\operatorname{tr}(\mathcal{R})} s^{\prime}$ then $s \theta \downarrow_{\mathcal{R}} s^{\prime} \theta$.

Proof. We prove these statements simultaneously, by induction on the minimum number of rewrite steps that witness the fact that the relation $s \rightarrow_{\operatorname{tr}(\mathcal{R})} s^{\prime}$, respectively $s \bigvee_{\operatorname{tr}(\mathcal{R})} s^{\prime}$, holds.

1. Let $(l \rightarrow r \Leftarrow c) \in \operatorname{tr}(\mathcal{R})$ with fresh variant $l^{\prime} \rightarrow r^{\prime} \Leftarrow c^{\prime}$, such that $s \rightarrow_{p, l^{\prime} \rightarrow r^{\prime} \Leftarrow c^{\prime}, \sigma} s^{\prime}$. This means, $\left.s\right|_{p}=l^{\prime} \sigma, s^{\prime}=s\left[r^{\prime} \sigma\right]_{p}$, and $t \sigma \backslash_{\operatorname{tr}(\mathcal{R})} t^{\prime} \sigma$ for every equation $t \approx t^{\prime}$ in $c^{\prime}$.

Let $\theta^{\prime}$ be a value substitution such that $\theta^{\prime}(x)=\theta(x)$ for all $x \in \operatorname{var}(s) \cup \operatorname{var}\left(s^{\prime}\right)$, and $\operatorname{evar}\left(l^{\prime} \rightarrow r^{\prime} \Leftarrow c^{\prime}\right) \subseteq \operatorname{dom}\left(\theta^{\prime}\right)$. Then $\left.s \theta\right|_{p}=l^{\prime} \sigma \theta^{\prime}, s^{\prime} \theta=s \theta\left[r^{\prime} \sigma \theta^{\prime}\right]_{p}$, and $t \sigma \theta^{\prime} \coprod_{\operatorname{tr}(\mathcal{R})} t^{\prime} \sigma \theta^{\prime}$ for all $t \approx t^{\prime}$ in $c^{\prime}$. Then $\operatorname{var}(t) \cup \operatorname{var}\left(t^{\prime}\right) \subseteq \operatorname{dom}\left(\sigma \theta^{\prime}\right)$ for all equations $t \approx t^{\prime}$ in $c^{\prime}$. By the induction hypothesis, $t \sigma \theta^{\prime} \downarrow_{\mathcal{R}} t^{\prime} \sigma \theta^{\prime}$ for all $t \approx t^{\prime}$ in $c^{\prime}$. Therefore, $s \theta \rightarrow_{p, l^{\prime} \rightarrow r^{\prime} \Leftarrow c^{\prime}, \sigma \theta^{\prime}} s^{\prime} \theta$, or simply $s \theta \rightarrow_{\mathcal{R}} s^{\prime} \theta$.

2. If $s \coprod_{\operatorname{tr}(\mathcal{R})} s^{\prime}$ then there exists $u \in \mathcal{T}(\mathcal{C}, \mathcal{X})$ such that $s \rightarrow_{\operatorname{tr}(\mathcal{R})}^{*} u$ and $s^{\prime} \rightarrow_{\operatorname{tr}(\mathcal{R})}^{*} u$. This implies $s \theta \rightarrow_{\operatorname{tr}(\mathcal{R})}^{*} u \theta$ and $s^{\prime} \theta \rightarrow_{\operatorname{tr}(\mathcal{R})}^{*} u \theta$. Note that all rewrite steps in a rewrite derivation remain valid if we instantiate the extra variables of rules with values. Therefore, we can assume without loss of generality that the rewrite derivations $s \theta \rightarrow_{\operatorname{tr}(\mathcal{R})}^{*} u \theta$ and $s^{\prime} \theta \rightarrow_{\operatorname{tr}(\mathcal{R})}^{*} u \theta$ consist of rewrite steps between values. By the induction hypothesis, every such rewrite step with respect to $\operatorname{tr}(\mathcal{R})$ is also a valid rewrite step with respect to $\mathcal{R}$. Thus, $s \theta \rightarrow_{\mathcal{R}}^{*} u \theta$ and $s^{\prime} \theta \rightarrow_{\mathcal{R}}^{*} u \theta$. Hence, $s \theta \downarrow_{\mathcal{R}} s^{\prime} \theta$.

Let $\operatorname{tr}(G)=s_{1} \approx t_{1} \& \& \ldots \& \& s_{n} \approx t_{n}$ be the system of equations produced from the system of equations $G=s_{1} \doteq t_{1} \& \& \ldots \& \& s_{n} \doteq t_{n}$ by replacing all occurrences of $\doteq / 2$ with $\approx / 2$. 
Lemma 1 implies that every complete set of answers of $G$ is also a set of answers of $\operatorname{tr}(G)$, but possibly incomplete. For example the set $A=\{\{x \mapsto t\} \mid t \in \mathcal{T}(\mathcal{C})\}$ is a complete set of answers for $G=x \doteq x$ with respect to $\mathcal{R}=\emptyset$, but it is incomplete for $\operatorname{tr}(G)=x \approx x$. The set $A$ can be computed with strategy INS for the OIS $\overline{\mathcal{R}}_{\text {eq }}$ which, in this trivial example, coincides with $\left\{\rho_{c} \mid c \in \mathcal{C}\right\}$.

A more desirable method would compute a complete set of answers for $\operatorname{tr}(G)$. For example, the set $A=\{\varepsilon\}$ where $\varepsilon$ is the empty substitution, is a complete set of computed answers for $\operatorname{tr}(G)=x \approx x$ with respect to $\mathcal{R}=\emptyset$. Besides being finite, this set gives a complete characterization of the set of solutions of $G=x \doteq x$ : Every solution of $G$ is a ground instantiation of $\sigma \in A$, that is, a value substitution of the form $\sigma \theta$ with $\sigma \in A$.

Thus, strategy INS can not be used to compute a complete set of answers for $\operatorname{tr}(G)$ w.r.t. $\operatorname{tr}(\mathcal{R})$. A first attempt to revise it is to transform $\operatorname{tr}(\mathcal{R})$ into the TRS

$$
\tilde{\mathcal{R}}:=\overline{\operatorname{tr}(\mathcal{R})}_{\text {eq }} \cup\{x \approx x \rightarrow \text { success }\} \text { where }
$$

$\overline{\operatorname{tr}(\mathcal{R})}$ is the OIS produced from the CB-CTRS $\operatorname{tr}(\mathcal{R})$ as indicated on page 101,

$$
\overline{\operatorname{tr}(\mathcal{R})}_{\mathrm{eq}}:=\overline{\operatorname{tr}(\mathcal{R})} \cup\left\{\rho_{c} \mid c \in \mathcal{C}\right\} \cup\{\text { success \&\& } x \rightarrow x\}
$$

and to impose the following restriction on the rewrite relation induced by $\tilde{\mathcal{R}}$ :

- A rewrite step $t \rightarrow_{p, x} \approx x \rightarrow$ success, $\sigma t^{\prime}$ is allowed iff $\sigma$ is a constructor substitution.

The following result is obvious: $\theta$ is a solution of $\operatorname{tr}(G)$ iff $\left.\theta\right|_{\operatorname{var}(\operatorname{tr}(G))}$ is a constructor substitution and $\operatorname{tr}(G) \theta \rightarrow_{\tilde{\mathcal{R}}}^{*}$ success.

Unfortunately, strategy $I N S_{\tilde{\mathcal{R}}}$ does not work well for $\tilde{\mathcal{R}}$ because $\tilde{\mathcal{R}}$ is not OIS. To fix this problem, we will make the following general assumptions:

- For every given sort $s$, we assume predefined an enumeration of all its constructors, and call it canonical order.

- $\mathcal{T}_{\approx}^{s}=\operatorname{branch}\left(x \approx y, 1, \mathcal{T}_{1}, \ldots, \mathcal{T}_{m}\right)$ is the definitional tree for the operation $\approx$ between terms of sort $s$, with the following structure:

$-c_{1} / n_{1}, \ldots, c_{m} / n_{m}$ is the enumeration of all constructors of sort $s$ in canonical order,

- Every $\mathcal{T}_{i}=\operatorname{branch}\left(\pi_{i}, 2, \mathcal{T}_{i, 1}, \ldots, \mathcal{T}_{i, m}\right)$ is the partial definitional tree of the linear pattern $\pi_{i}=c_{i}\left(x_{1}, \ldots, x_{n_{i}}\right) \approx y$ where

$$
\mathcal{T}_{i, j}= \begin{cases}\operatorname{exempt}\left(c_{i}\left(x_{1}, \ldots, x_{n_{i}}\right) \approx c_{j}\left(y_{1}, \ldots, y_{n_{j}}\right)\right) & \text { if } i \neq j \\ \operatorname{rule}\left(\rho_{c}\right) & \text { if } i=j .\end{cases}
$$

Next, we specialize INS to work for $\tilde{\mathcal{R}}$ in the following way:

1. $I N S_{\tilde{\mathcal{R}}}\left(t_{1} \approx t_{2}\right)$ is either

(a) $\left\langle\lambda, x \approx x \rightarrow\right.$ success, $\left.\left\{t_{1} \rightarrow t_{2}\right\}\right\rangle$ if $t_{1}, t_{2} \in \mathcal{X}$ and $t_{1} \neq t_{2}$.

(b) $\langle\lambda, x \approx x \rightarrow$ success, \{\}$\rangle$ if $t_{1}=t_{2} \in \mathcal{X}$.

(c) $\varphi_{\tilde{\mathcal{R}}}\left(t_{1} \approx t_{2}, \mathcal{T}_{\approx}^{s}\right)$ otherwise, where $s$ is the sort of $t_{1}$.

2. $I N S_{\tilde{\mathcal{R}}}(t)$ is defined like $I N S_{\overline{\mathcal{R}}}(t)$ in all other cases.

We are ready now to prove that the narrowing strategy $I N S_{\tilde{R}}$ is sound and complete. Let's assume $G$ is of the form $s_{1} \approx t_{1} \& \& \ldots \& \& s_{n} \approx t_{n}$, or success \&\& $s_{1} \approx t_{1} \& \& \ldots \& \& s_{n} \approx t_{n}$.

Lemma 2 (Soundness). If $\langle$ success, $\theta\rangle \in A n s_{\tilde{\mathcal{R}}}^{I N S_{\tilde{\mathcal{R}}}}(G)$ then $\theta$ is a solution of $G$. 
Proof. By the definition of $A n s_{\tilde{\mathcal{R}}}^{I N S_{\tilde{\mathcal{R}}}}(G)$, for every $\langle$ success, $\theta\rangle \in A n s_{\tilde{\mathcal{R}}}^{I N S_{\tilde{\mathcal{R}}}}(G)$ there exists a narrowing derivation

$$
G=G_{0} \rightsquigarrow q_{1}, \rho_{1}, \theta_{1} G_{1} \rightsquigarrow q_{2}, \rho_{2}, \theta_{2} \ldots \rightsquigarrow q_{n}, \rho_{n}, \theta_{n} G_{n}=\text { success }
$$

where $\rho_{i}$ are fresh variants of rewrite rules from $\tilde{\mathcal{R}}$ and $\left.\theta_{i}\right|_{\operatorname{var}\left(G_{i-1}\right)}$ are constructor substitutions, for all $1 \leq i \leq n$, such that $\theta=\theta_{1} \theta_{2} \ldots \theta_{n}$. It follows that $G \theta \rightarrow_{\mathcal{R}}^{*}$ success, thus $\theta$ is solution of $G$.

Lemma 3 (Completeness). If $\theta$ is a solution of $G$ then there exists $\left\langle\right.$ success, $\left.\theta^{\prime}\right\rangle \in \operatorname{Ans}_{\tilde{\mathcal{R}}}^{I N S}(G)$ such that $\theta^{\prime} \leq \theta$.

\section{A rewrite-based strategy}

In this section we introduce our second contribution: a rewrite-based strategy capable to simulate $I N S_{\tilde{\mathcal{R}}^{-n a r r o w i n g}}$ derivations. The insight behind the design of this strategy is based on following observation: Every successful search of a narrowing step $\langle p, l \rightarrow r, \sigma\rangle$ with strategy $I N S_{\tilde{R}}$ can be decomposed into a sequence of elementary steps of three kinds:

T1. Binding steps, which bind a variable to a constructor term. To this category belong case 1.(a).i from the unspecialized definition of $I N S_{\tilde{\mathcal{R}}}$, and case 1.(a) from the definition of the specialized version.

T2. Lookup steps, which simply traverse the term in depth to detect a suitable narrowing position. To this category belong cases 1.(a).ii, 1.(a).iii, and 3 from the unspecialized definition of $I N S_{\tilde{\mathcal{R}}}$.

T3. Final search steps, which detect the possibility to rewrite an instance of the traversed term with a rewrite rule at a certain position. To this category belong cases 1.(b) from the unspecialized definition of $I N S_{\tilde{\mathcal{R}}}$, and case 1.(b) in the definition of the specialized version.

Starting from this observation, we can "sequentialize" strategy INS to stop as soon as it performs the first step of type T1 or type T3, and return the corresponding variable binding (if the step type is T1) or position/rule (if the step type is T3). We call IRS the "sequentialized" version of INS, because it is intended to stand for Inductively sequential $\mathbf{R}$ ewriting $\mathbf{S}$ trategy:

- When it returns a binding of the form $x \rightarrow t$, it indicates the need to perform maximum parallel rewriting with the rewrite rule $x \rightarrow t$ as if $x$ were a constant. Note that such a parallel rewrite step has the same effect as applying the substitution $\{x \rightarrow t\}$.

- When it returns a pair $\langle p, l \rightarrow r\rangle$, it indicates the need to perform the rewrite step with rule $l \rightarrow r$ at position $p$.

To ensure the fact that IRS simulates INS, we must guarantee that every call of the IRS strategy proceeds from the place where the previous call of IRS stopped the computation of an INS-step. Based on these considerations, we came up with the following definition of $\operatorname{IR} S_{\tilde{\mathcal{R}}}$ :

- $\operatorname{IR} S_{\tilde{\mathcal{R}}}(x \approx y)=x \rightarrow y$ if $x, y \in \mathcal{X}$ and $x \neq y$.

$\operatorname{IR} S_{\tilde{\mathcal{R}}}(y \approx y)=\{\langle\lambda, x \approx x \rightarrow$ success $\rangle\}$ if $y \in \mathcal{X}$.

$I R S_{\tilde{\mathcal{R}}}\left(t_{1} \approx t_{2}\right)=D T_{\tilde{\mathcal{R}}}\left(t_{1} \approx t_{2}, \mathcal{T}_{\approx}^{s}\right)$ if $\left\{t_{1}, t_{2}\right\} \not \subset \mathcal{X}$ and $s$ is the sort of $t_{1}$. (The auxiliary method $D T_{\tilde{\mathcal{R}}}(t)$ is defined below.) 
- Otherwise, there exists a leftmost outermost position $p \neq \lambda$ of $t$ such that $\left.t\right|_{p}=f\left(t_{1}, \ldots, t_{n}\right)$ with $f \in \mathcal{F}$. Let $A=I R S_{\tilde{\mathcal{R}}}\left(\left.t\right|_{p}\right)$. Then

$$
I R S_{\tilde{\mathcal{R}}}(t)= \begin{cases}\{\langle p \cdot q, \rho\rangle \mid\langle q, \rho\rangle \in A & \text { if } A \neq \emptyset \text { is set of pairs, } \\ A & \text { otherwise. }\end{cases}
$$

The auxiliary method $D T_{\overline{\mathcal{R}}}(t, \mathcal{T})$ is defined as follows:

1. If $\mathcal{T}=\operatorname{branch}\left(\pi, p, \mathcal{T}_{1}, \ldots, \mathcal{T}_{k}\right)$, let $s$ be the sort of $\left.t\right|_{p}$ and $c_{1} / n_{1}, \ldots, c_{k} / n_{k}$ be the data constructors of sort $s$ in canonical order. We distinguish three subcases:

(a) If $\left.t\right|_{p}=x \in \mathcal{X}$, let $\sigma_{i}=\left\{x \rightarrow c_{i}\left(x_{1}, \ldots, x_{n_{i}}\right)\right\}$ be substitutions with $x_{1}, \ldots, x_{n_{i}}$ fresh variables of appropriate sorts, for all $1 \leq i \leq n$. Then

$D T_{\tilde{\mathcal{R}}}(t, \mathcal{T})=\left\{x \rightarrow c_{i}\left(x_{1}, \ldots, x_{n_{i}}\right) \mid 1 \leq i \leq k\right\}$.

(b) If $\left.t\right|_{p}=c_{i}\left(s_{1}, \ldots, s_{n_{i}}\right)$ then $D T_{\tilde{\mathcal{R}}}(t, \mathcal{T})=D T_{\tilde{\mathcal{R}}}\left(t, \mathcal{T}_{i}\right)$.

(c) If $\left.t\right|_{p}=g\left(s_{1}, \ldots, s_{m}\right)$ with $g \in \mathcal{F}$, let $A=D T_{\tilde{\mathcal{R}}}\left(\left.t\right|_{p}, \mathcal{T}_{g}\right)$. Then

$$
D T_{\tilde{\mathcal{R}}}(t, \mathcal{T})= \begin{cases}\{\langle p \cdot q, \rho\rangle \mid\langle q, \rho\rangle \in A\} & \text { if } A \neq \emptyset \text { is set of pairs } \\ A & \text { otherwise. }\end{cases}
$$

2. If $\mathcal{T}=\operatorname{rule}\left(\pi, \pi \rightarrow r_{1}\right.$ ? $\ldots$ ? $\left.r_{k}\right)$ then $D T_{\tilde{\mathcal{R}}}(t, \mathcal{T})=\left\{\left\langle\lambda, \pi \rightarrow r_{i}\right\rangle \mid 1 \leq i \leq k\right\}$.

3. Otherwise, $\mathcal{T}=\operatorname{exempt}(\pi)$ and $D T_{\tilde{\mathcal{R}}}(t, \mathcal{T})=\emptyset$.

An IRS-compliant rewrite step of a term $t$ w.r.t. $\tilde{\mathcal{R}}$ is a relation $t \Rightarrow_{\sigma} t^{\prime}$ which holds if either (1) $\langle p, \rho\rangle \in I R S_{\tilde{\mathcal{R}}}(t)$. In this case $\sigma=\{\}$ and $t^{\prime}$ is the result of the rewrite step $t \rightarrow_{p, \rho} t^{\prime}$, or (2) $x \rightarrow t$ is a substitution rule from $\operatorname{IR} S_{\tilde{\mathcal{R}}}(t)$. In this case $\sigma=\{x \rightarrow t\}$ and $t^{\prime}$ is the result of maximal parallel rewriting with the rewrite rule $x \rightarrow t$, as if $x$ were a constant. An IRS-compliant rewrite derivation of a term $t$ w.r.t. $\tilde{\mathcal{R}}$ is a sequence $t \Rightarrow_{\sigma_{1}} t_{1} \Rightarrow_{\sigma_{2}} \ldots \Rightarrow_{\sigma_{n}} t_{n}$, abbreviated $t \Rightarrow_{\sigma}^{*} t_{n}$ where $\sigma=\left.\left(\sigma_{n} \circ \ldots \circ \sigma_{2} \circ \sigma_{1}\right)\right|_{\operatorname{var}(t)}$. For a given system of equations $G$, the set of answers computed by our rewrite-based strategy is defined to be

$$
A n s_{\tilde{\mathcal{R}}}^{I R S_{\tilde{\mathcal{R}}}}(G)=\left\{\langle\text { success }, \sigma\rangle \mid G \Rightarrow_{\sigma}^{*} \text { success }\right\} .
$$

Theorem 1. Let $\mathcal{R}$ be an OIS. Then $\operatorname{Ans}_{\tilde{\mathcal{R}}}^{I R S_{\tilde{\mathcal{R}}}}(G)=\operatorname{Ans}_{\tilde{\mathcal{R}}}^{I N S_{\tilde{\mathcal{R}}}}(G)$.

Since strategy $I N S_{\tilde{\mathcal{R}}}$ is sound and complete, we conclude that strategy $I R S_{\tilde{\mathcal{R}}}$ is sound and complete too.

\section{Concluding remarks}

Overlapping inductively sequential TRSs with extra variables have emerged as a convenient core language for FLP computations. Unfortunately, the INS strategy can not be applied directly to solve systems of equations where equality is interpreted as reducibility to a common constructor term. The reason for this is that there is no way to define this kind of equality with rewrite rules of this kind. We have identified a simple way to overcome this limitation: to extend the underlying OIS with the nonlinear rewrite rule $x \approx x \rightarrow$ success, and to impose restrictions on the way it is used in rewrite and narrowing derivations. By exploring this idea, we have identified a simple adjustment of strategy $I N S$, which is sound and complete for our generalized interpretation of equality (Section 3). Moreover, we found a way to simulate the narrowing computations of interest to us with a strategy for rewrite derivations (Section 4). The efficiency of both strategies stems from the fact that both of them are controlled by definitional trees. 


\section{Acknowledgments}

Temur Kutsia has been partially supported by the Austrian Science Fund (FWF) under the projects P 24087-N18 and P 28789-N32. Besik Dundua has been partially supported by the Shota Rustaveli National Science Foundation under the grants FR/325/4-120/14, YS/10/11811/15, YS15_2.1.2_70.

\section{References}

[1] Sergio Antoy. Definitional trees. In Proceedings of the 3rd International Conference on Algebraic and Logic Programming, pages 143-157. Springer LNCS, 1992.

[2] Sergio Antoy. Optimal non-deterministic functional logic computations. In Michael Hanus, Jan Heering, and Karl Meinke, editors, Algebraic and Logic Programming, volume 1298 of Lecture Notes in Computer Science, pages 16-30. Springer Berlin Heidelberg, 1997.

[3] Sergio Antoy. Constructor-based conditional narrowing. In Proceedings of PPDP 2001, pages 199-206. ACM Press, 2001.

[4] Sergio Antoy, Rachid Echahed, and Michael Hanus. A needed narrowing strategy. In Journal of the ACM, pages 268-279. ACM Press, 1994.

[5] Sergio Antoy, Rachid Echahed, and Michael Hanus. Parallel Evaluation Strategies for Functional Logic Languages. In Proceedings of the 14th International Conference on Logic Programming (ICLP'97), pages 138-152. MIT Press, 1997.

[6] Sergio Antoy and Michael Hanus. Overlapping rules and logic variables in functional logic programs. In 22nd International Conference on Logic Programming, pages 87-101. Springer LNCS, 2006.

[7] Sergio Antoy and Michael Hanus. Functional logic programming. Communications of the ACM, 53(4):74-85, 2010.

[8] J.C. Gonzalez-Moreno, M.T. Hortala-Gonzalez, F.J. Lopez-Fraguas, and M. Rodriguez-Artalejo. An approach to declarative programming based on a rewriting logic. The Journal of Logic Programming, 40(1):47-87, 1999.

[9] Michael Hanus, Salvador Lucas, and Aart Middeldorp. Strongly sequential and inductively sequential term rewriting systems. Information Processing Letters, 67(1):1-8, 1998.

[10] Jean-Marie Hullot. Canonical forms and unification. In Wolfgang Bibel and Robert Kowalski, editors, 5th Conference on Automated Deduction Les Arcs, France, July 8-11, 1980, volume 87 of Lecture Notes in Computer Science, pages 318-334. Springer Berlin Heidelberg, 1980.

[11] Tetsuo Ida, Mircea Marin, and Taro Suzuki. Higher-Order Lazy Narrowing Calculus: A Solver for Higher-Order Equations. In Computer Aided Systems Theory - EUROCAST 2001-Revised Papers, pages 479-493, London, UK, 2001. Springer-Verlag.

[12] Mircea Marin. Functional Logic Programming with Distributed Constraint Solving. PhD thesis, RISC-Linz Institute, Schloss Hagenberg, Austria, 2000.

[13] Mircea Marin and Aart Middeldorp. New completeness results for lazy conditional narrowing. In Proceedings of the 6th ACM SIGPLAN Conference on Principles and Practice of Declarative Programming, pages 120-131, Verona, 2004. ACM Press.

[14] Aart Middeldorp and Satoshi Okui. A deterministic lazy narrowing calculus. Journal of Symbolic Computation, 25(6):733-757, 1998.

[15] Aart Middeldorp, Satoshi Okui, and Tetsuo Ida. Lazy narrowing: Strong completeness and eager variable elimination. Theoretical Computer Science, 167(1,2):95-130, 1996.

[16] Aart Middeldorp, Taro Suzuki, and Mohamed Hamada. Complete selection functions for a lazy conditional narrowing calculus. J. Functional and Logic Programming, 2002(3), March 2002.

[17] Christian Prehofer. Solving higher-order equations: from logic to programming. Birkhauser, 1998. 